\title{
Incidence of meningitis in blood culture proven neonatal sepsis in a pediatric tertiary care hospital in Bangalore
}

\author{
Mahantesh. $\mathbf{S}^{1}$, Manasa.S ${ }^{2}$, Niranjan.H.S ${ }^{3}$ \\ ${ }^{1}$ Dr. Mahantesh. S, Associate Professor, Department of Microbiology, ${ }^{2}$ Dr. Manasa. S, Scientist B, IGICH Bangalore, \\ ${ }^{3}$ Dr. Niranjan. H.S, Associate Professor, Department of Pediatrics, IGICH Bangalore, Karnataka, India.
}

Address for Correspondence: Dr. Manasa. S, Department of Microbiology, IGICH Bangalore, Email: manasabharadwaj86@gmail.com

\begin{abstract}
Objectives: Neonatal bacterial meningitis continues to be an important cause of mortality and morbidity. Contributing factors to such mortality and morbidity include our incomplete knowledge on the pathogenesis of how meningitiscausing bacteria penetrate the blood brain barrier, emergence of antimicrobial resistance, and difficulty in early diagnosis of meningitis. An early empiric antibiotic treatment is critical for the management of neonates with bacterial meningitis, but early recognition of neonatal meningitis continues to be a challenge. The concordance between blood, and CSF cultures may affect management strategies for sepsis and meningitis so this study was carried out. Materials and Methods: This is a retrospective study done over a period of 12 months from January 2016 to December 2016. Blood culture results were compared with results of CSF cultures and CSF parameters like white blood cells, glucose, and protein to establish the concordance of these values in culture-proven meningitis. Results: Out of 616 CSF cultures sent, culture positives were seen in $47(7.6 \%)$ neonates. Of the 47 patients with meningitis, $40(85.1 \%)$ had a documented blood culture and $7(14.8 \%)$ was contaminants. In neonates with both positive blood and CSF cultures, the organisms isolated were discordant in $2(4.2 \%)$ of 40 cases. The common organisms isolated was Group B streptococci 14 (29\%), Escherichia coli $10(21 \%)$, followed by CONS $7(14.8 \%)$, staphylococcus aureus $7(14.8 \%)$ and Klebsiella species 2 (4.2\%). Conclusion: In this study Neonatal meningitis frequently occurs in the absence of bacteremia and in the presence of normal CSF parameters. No single CSF value can reliably exclude the presence of meningitis in neonates. The CSF culture is critical to establishing the diagnosis of neonatal meningitis.
\end{abstract}

Key words: Meningitis; Blood culture; Cerebrospinal fluid parameters; Bacteremia.

\section{Introduction}

Meningitis is associated with high mortality and longterm complications among survivors in the neonatal period [1-3]. Adequate treatment relies on proper diagnosis, and only cerebrospinal fluid (CSF) culture can definitively diagnose meningitis [1,4-8]. When evaluating an infant for sepsis, many clinicians choose to obtain a blood culture and await a positive result before performing a lumbar puncture. This strategy underestimates the incidence of meningitis; furthermore, up to $50 \%$ of very low birth weight [8].

The concordance between blood, urine and CSF cultures may affect management strategies for sepsis

Manuscript received: $1^{\text {st }}$ June 2017

Reviewed: $11^{\text {th }}$ June 2017

Author Corrected: $20^{\text {th }}$ June 2017

Accepted for Publication: $26^{\text {th }}$ June 2017 and meningitis. Meningitis in the neonate is classified as early- or late-onset depending on the time after birth when it occurs. The majority $(61.4 \%)$ of late-onset sepsis cases are caused by Gram-positive organisms [9]. Prior studies have found $39 \%$ of blood and CSF cultures to be concordant with Gram-positive organisms compared with $16 \%$ of blood and urine cultures $[10,11]$. However, the relationship between individual organisms present in blood and CSF cultures, as well as between concordance and the timing of blood and CSF cultures, have not been fully described.

We sought to determine the concordance of organisms between blood and CSF cultures and to evaluate predictors of concordance adjusting for markers of severity of illness. 


\section{Materials and Methods}

Source of data: This is a retrospective study done over a period of 12 months from January 2016 to December 2016. The study will be conducted in the department of Microbiology, IndiraGandhi institute of child health.

The data was analysed, neonates having positive CSF culture was paired with blood culture and the other criteria of meningitis like CSF cell count, protein and glucose was also compared.

\section{Inclusion criteria}

- We included positive blood cultures in the analysis if they could be paired with a CSF culture obtained in the same infant on the same day as the blood culture or up to three days before or three days after the positive blood culture.

- If multiple blood cultures could be paired with the same CSF culture, they were each counted as separate blood-CSF culture pair.

- If multiple CSF cultures could be paired with the same blood culture, we kept, in order of preference, a positive CSF culture over a negative CSF culture, a CSF culture obtained on the same day over a CSF culture obtained before or after the blood culture, and then a CSF culture obtained closest in time before or after the blood culture.

\section{Exclusion criteria}

- We excluded blood cultures positive for organisms considered contaminants, including non-speciated streptococci, Bacillus sp., Corynebacterium sp., diphtheroids sp., Gram-positive rods (not including Listeria sp.), Lactobacillus sp., Micrococcus sp., Stomatococcus sp. and Bacteroides sp.

- All blood cultures that could not be paired with a CSF culture and all CSF cultures that could not be paired with a blood culture were excluded from the analysis.

Methodology: The blood and CSF which were obtained from the patient was immediately transferred to the microbiology lab. The specimens will be processed immediately first by inoculating onto media culture and then direct smear examination by Gram's Stain to avoid contamination.

The samples will be processed by inoculating onto the following media: i. 5\% sheep blood agar and MacConkey agar, incubated at $37^{\circ} \mathrm{C}$ aerobically for $24 \mathrm{hrs}$.

ii. Chocolate agar, incubated at $37^{\circ} \mathrm{C}$ in the presence of $5-10 \%$ of $\mathrm{CO}_{2}$ in a candle jar for $24 \mathrm{hrs}$.

iii. Brain heart infusion broth $5-10 \mathrm{ml}$, incubated at $37^{0} \mathrm{C}$ aerobically

iv. Anaerobic culture will be done using Robertson's cooked meat media and incubated at $37^{\circ} \mathrm{C}$.

The culture on blood agar, chocolate agar will be incubated for 48 hours and if there is no growth, these media will be incubated for 8 more days to allow the growth of slow growing or fastidious organisms.

The cultures for anaerobic bacteria will be incubated for 2 weeks for any evidence of growth. The control strains used were Escherichia Coli ATCC 25922, Pseudomonas aeruginosa, ATCC 27853 and Staphylococcus aureus ATCC 25923. Controls were put up as recommended.

Direct smear: Two smears were prepared on a sterile glass slides and air dried. Gram's stain was done and smears were examined for the presence of pus cells and organisms.

Criteria for laboratory confirmed growth:

1) Growth on a single media correlating with direct microscopy findings.

2) Growth of the bacteria from the CSF or blood on two or more of the inoculated media.

3) Repeated isolation of the same organisms from two or more specimens of the patients.

4) Semi-confluent growth on one or more solid media at the inoculation site.

5) Any growth in anaerobic media.

Based on the gram stain of the culture the isolates were identified by using standard techniques.

All the cultures was subjected for antimicrobial susceptibility by means of agar disc diffusion method of Kirby Bauer according to the guidelines of clinical and laboratory standards. When multiple positive blood cultures with the same organism were obtained within a 21-day period, they were considered to be a common 
Research Article

infectious episode, and only the first positive culture was retained for analysis. Multiple CSF cultures within the same 21-day period were also considered to be a common infectious episode, and only the first positive culture was retained for analysis. Blood and CSF cultures positive with $>1$ organism were treated as separate positive cultures. If 1 of the organisms was considered a contaminant, we counted only the true organism as a positive culture and ignored the contaminant. We defined blood-CSF culture concordance as the growth of the same organism in the blood and CSF of the blood-CSF culture pair. We defined blood-CSF culture discordance as either the growth of an organism in the blood culture but not the CSF culture, or the growth of two different organisms in each one of the two cultures of each pair. We also examined group B streptococcal (GBS) concordance in early- and late-onset GBS sepsis.

We determined concordance for early-onset $(<3$ days of life) as well as late-onset ( $\geq 3$ days of life) GBS sepsis in infants with a GBS-positive blood culture

\section{Results}

In our study conducted Out of 616 CSF cultures sent only 47(7.6\%) had growth and 569(92.3\%) had no growth.

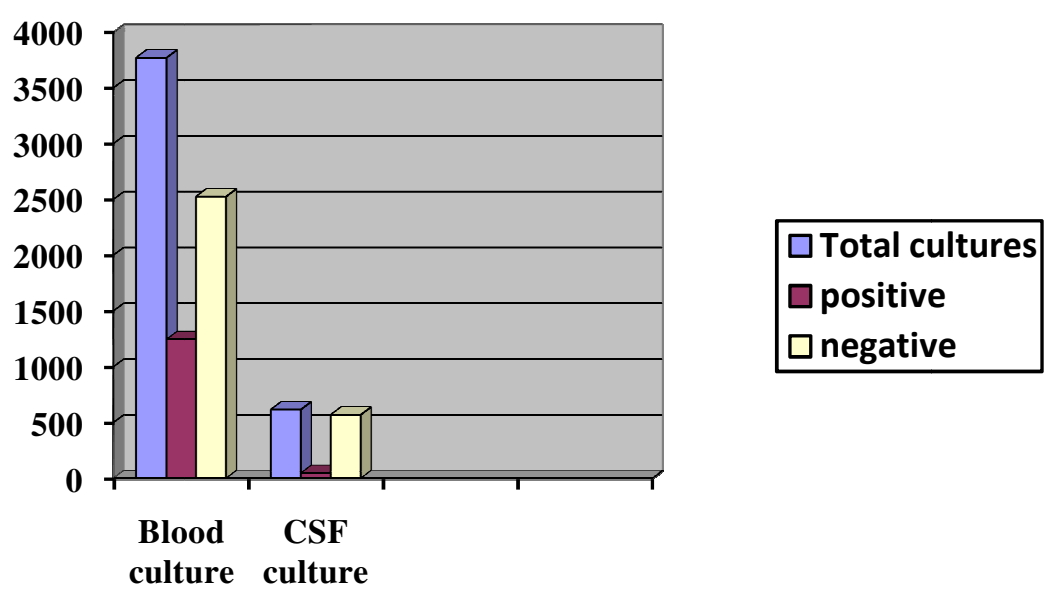

FIG-1: Total Blood and CSF cultures and their positive and negative culture

Out of 3770 blood cultures sent $1246(33 \%)$ had positive blood cultures. Out of 616 CSF cultures sent, culture positives was seen in $47(7.6 \%)$ neonates. Of the 47 patients with meningitis, 40(85.1\%) had a documented blood culture and $7(14.8 \%)$ was contaminants. In neonates with both positive blood and CSF cultures, the organisms isolated were discordant in $2(4.2 \%)$ of 40 cases. The common organisms isolated was Group B streptococci 14(29\%), Escherichia coli 10(21\%), followed by CONS 7(14.8\%), staphylococcus aureus 7(14.8\%) and Klebsiella species 2(4.2\%).
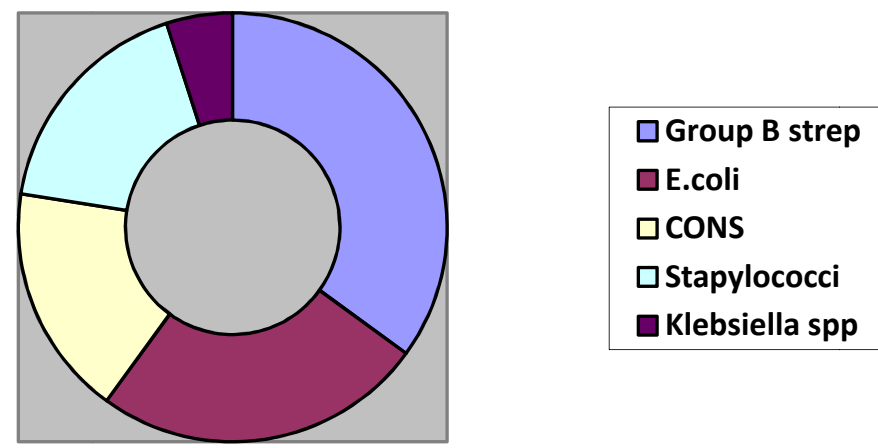

Fig-2: Distribution of Various organisms isolated

In the staph aureus the major organism was methicillin resistant staphylococcus aureus than the methicillin sensitive staphylococcus organisms. 
Research Article

The results of the susceptibility tests of the gram negative bacterial isolates from CSF samples to the commonly used antibiotics showed resistance as follows : cefazolin $(76.5 \%)$ ampicillin $(71.8 \%)$, amoxyclav ( $65.2 \%)$, ceftriaxone (68.5\%), piperacillin (62.9\%), ceftazidime (47.5\%), amikacin (45.2\%) and ciprofloxacin (66.9\%). Imipenem (48\%)and meropenum was $(35 \%)$ showed moderate resistance.

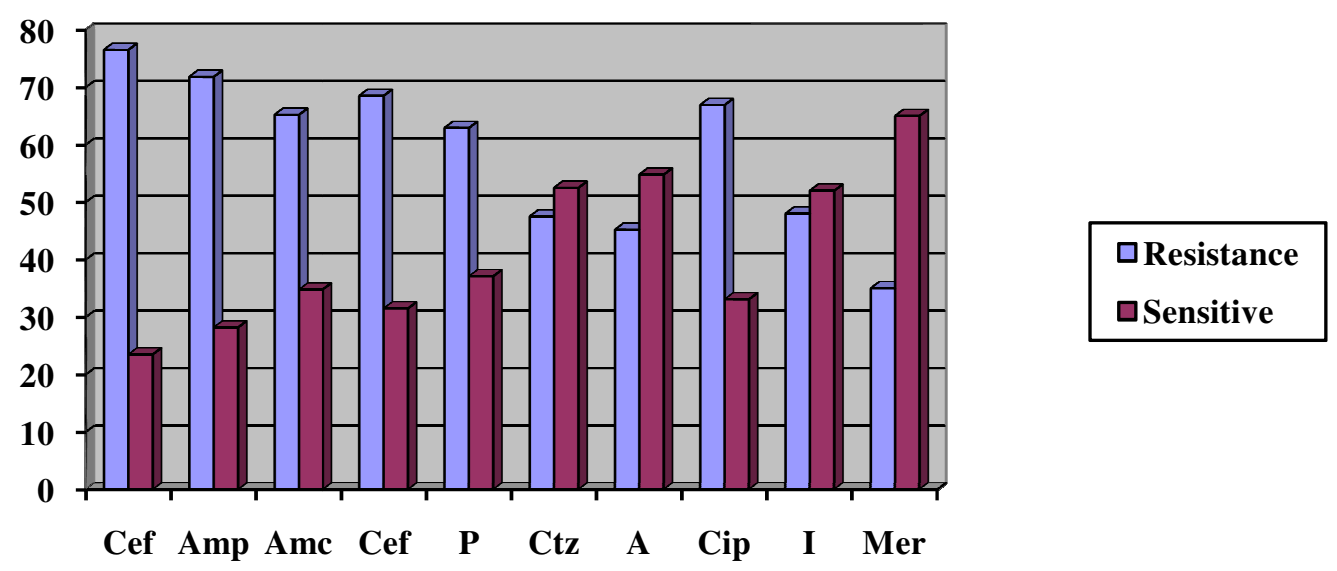

Fig-3: Sensitivity of Gram negative organisms

The gram positive organism showed resistance as follows : amoxiclav $(60 \%)$, cefuroxime $(40 \%)$, chloramphenicol (20\%), ciprofloxacin $(20 \%)$, clindamycin $(50 \%)$, cotrimoxazole $(60 \%)$ to erythromycin( $20 \%)$, gentamycin $(40 \%)$ tetracycline (12\%) and they are completely sensitive to Vancomycin and linezolid.

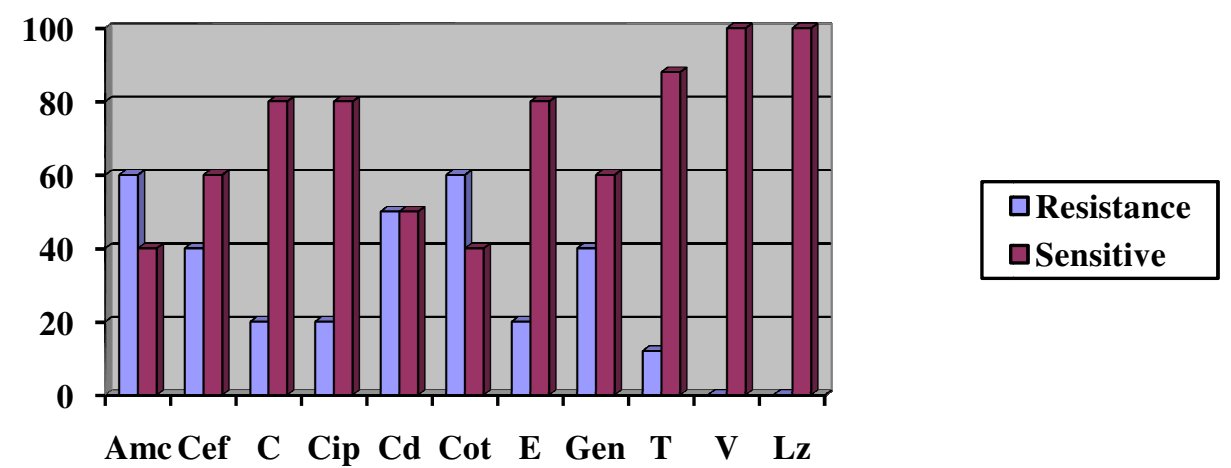

Fig-4: Sensitivity of Gram positive organisms

In neonates with both positive blood and CSF cultures, the organisms isolated were discordant in $2(5 \%)$ of 40 cases. In each case, the CSF pathogen required different antimicrobial therapy than the blood pathogen. For culture-proven meningitis, CSF WBC counts of $>0$ cells per $\mathrm{mm} 3$ had sensitivity at $97 \%$ and specificity at $10 \%$. CSF WBC counts of $>21$ cells per $\mathrm{mm} 3$ had sensitivity at $81 \%$ and specificity at $84 \%$. Culture-proven meningitis was not diagnosed accurately by CSF glucose or by protein.

\section{Discussion}

Neonatal sepsis remains one of the leading causes of morbidity and mortality both among term and preterm infants. [12]. Although advances in neonatal care have improved survival and reduced complications in preterm infants, sepsis still contributes significantly to mortality and morbidity among very-low-birth-weight (VLBW, $<1500 \mathrm{~g}$ ) infants in Neonatal Intensive Care Units (NICUs) [13-17].
The signs and symptoms of neonatal sepsis are nonspecific [15]. These include fever or hypothermia, respiratory distress including cyanosis and apnoea, feeding difficulties, lethargy or irritability, hypotonia, seizures, bulging fontanel, poor perfusion, bleeding problems, abdominal distension, hepatomegaly, gauiacpositive stools, unexplained jaundice, or more importantly, "just not looking right" [16,17]. Infants 
Research Article

with hypoxia-acidosis may gasp in-utero and lead to pneumonia and meconium aspiration [18]. The incidence of neonatal sepsis or bacteremia in asymptomatic infants is low, but not negligible. Voora et al. reported a $1 \%$ prevalence of fever in term newborns with $10 \%$ of the febrile $\left(\geq 37.8{ }^{\circ} \mathrm{C}\right.$ rectal or core body temperature) infants having culture-proven sepsis [19].

While term newborns were described as being more likely to react to a bacterial infection with fever, preterm newborns were more likely to react with hypothermia, because of transitional difficulty with temperature control especially in the first 2 days [20,21]. In contrast, the lack of clinical relevance of body temperature in diagnosing sepsis later in preterm infants might be attributable to the use of incubators [22]. However, neonates with core body temperature elevation sustained for more than $1 \mathrm{~h}$, not due to environmental causes and greater than $39{ }^{\circ} \mathrm{C}$ are more likely to have bacteremia, meningitis, pneumonia, and also associated with viral disease, particularly herpes simplex encephalitis and therefore evaluation should include lumber puncture. [23].

Respiratory distress including tachypnea, grunting, nasal flaring, and retraction of respiratory muscles can be the sole manifestation of sepsis with or without pneumonia and can be confused with transient tachypnoea of newborn initially. Rapid clinical deterioration ensues unless prompt antibiotic management is started in neonates with sepsis. Neonatal sepsis can be complicated by metastatic foci of infection, disseminated intravascular coagulation, congestive heart failure and shock [24].

In our study group, we observed that blood-CSF culture concordance was associated with the presence of Gramnegative organisms, presence of a VP shunt, and timing of the CSF culture on the same day as the blood culture. We also observed increased concordance in cultures from infants exposed to CSF-penetrating antibiotics prior to blood-CSF culture pairing. Because meningitis in the infant is often due to the spread of pathogens from the blood into the CSF, and organisms frequently associated with peritoneal infection are Gram-negative rods including E. coli, it is possible that these pathogens can cause meningitis in several ways:

They may spread from a local peritoneal infection to the blood and continue to spread to the ventricles, or they may spread from the peritoneum into the ventricles of the brain through the shunt, leading to meningitis. Alternatively, it is possible that infection starts within the shunt itself and spreads to the blood stream, causing sepsis due to structural abnormalities in the shunts. Gram-positive organisms were cultured more frequently in our study than other organisms, i.e group B streptococci, which is consistent with study by Jean-Baptiste et al and Stoll et al. [11,25].

The overall incidence of culture-positive meningitis, as well as the distribution of organisms in our study is similar to stoll BJ et al [26,27].

We found that the proportion of concordant CSF and blood cultures was four times higher when the blood and CSF cultures were obtained on the same day compared with when the CSF was obtained before or after the blood culture. This demonstrates the importance of obtaining the CSF at the time of the sepsis evaluation to most accurately diagnose and treat potential meningitis in an infant. CSF should continue to be collected at the time of sepsis evaluation for diagnosis of meningitis confirmation and guidance of correct treatment, if the infant is able to withstand the procedure.

In our study we found that CSF culture positivity is more important than the other factors like CSF cell count, protein content and the glucose this is in concordance to the study by Garges HP et al. [11] which showed CSF WBC counts of $>0$ cells per mm 3 had sensitivity at $97 \%$ and specificity at $11 \%$. CSF WBC counts of $>21$ cells per $\mathrm{mm}$ had sensitivity at $79 \%$ and specificity at $81 \%$.

\section{Conclusion}

In this study, we found that the presence of a VP shunt, increased severity of illness, and obtaining blood and CSF cultures on the same day were all related to an increase in the possibility of a concordant blood-CSF culture pair. This concordance also varied depending on the specific organism. Overall, obtaining a lumbar puncture in a timely manner in these infants is essential. If more lumbar punctures are obtained on the same day and even at the same time as the blood culture, it is possible that more infants might be diagnosed with meningitis and treated accordingly.

Funding: Nil, Conflict of interest: None initiated, Permission from IRB: Yes 


\section{References}

1. Harvey D, Holt DE, Bedford H. Bacterial meningitis in the newborn: a prospective study of mortality and morbidity. Semin Perinatol.1999 Jun;23(3):218-25.

2. Stoll BJ, Hansen NI, Adams-Chapman I, et al. National Institute of Child Health and Human Development Neonatal Research Network. Neuro developmental and growth impairment among extremely low-birth-weight infants with neonatal infection. JAMA. 2004;292:2357-2365.

3. Benjamin DK, DeLong E, Cotten CM, et al. Mortality following blood culture in premature infants: increased with Gram-negative bacteremia and candidemia, but not Gram-positive bacteremia. J Perinatol. 2004; 24: 175-180. doi: 10. 1038/sj.jp. 7211068 .

4. Garges HP, Moody MA, Cotten CM, et al. Neonatal meningitis: what is the correlation among cerebrospinal fluid cultures, blood cultures, and cerebrospinal fluid parameters? Pediatrics. 2006;117:1094-1100. doi:10. 1542/ peds.2005-1132

5. de Louvois J. Acute bacterial meningitis in the newborn. J Antimicrob Chemother. 1994 Aug;34 Suppl A:61-73.

6. Johnson CE, Whitwell JK, Pethe K, Saxena K, Super DM. Term newborns who are at risk for sepsis: are lumbar punctures necessary? Pediatrics. 1997 Apr;99 (4):E10.

7. Wiswell TE, Baumgart S, Gannon CM, Spitzer AR. No lumbar puncture in the evaluation for early neonatal sepsis: will meningitis be missed? Pediatrics. 1995 Jun; 95(6):803-6.

8. Stoll BJ, Hansen N, Fanaroff AA, et al. To tap or not to tap: high likelihood of meningitis without sepsis among very low birth weight infants. Pediatrics. 2004; 113:1181-1186.

9. Hornik CP, Fort P, Clark RH, et al. Early and late onset sepsis in very-low-birth-weight infants from a large group of neonatal intensive care units. Early Hum Dev. 2012;88(suppl 2):S69-S74.

10. Downey LC, Benjamin DK Jr, Clark RH, et al. Urinary tract infection concordance with positive blood and cerebrospinal fluid cultures in the neonatal intensive care unit. J Perinatol. 2013;33:302-306.

11.Garges HP, Moody MA, Cotten CM, Smith PB, Tiffany KF, Lenfestey R, Li JS, Fowler VG Jr, Benjamin DK Jr. Neonatal meningitis: what is the correlation among cerebrospinal fluid cultures, blood cultures, and cerebrospinal fluid parameters? Pediatrics. 2006 Apr;117(4):1094-100.

12. Camacho-Gonzalez A, Spearman PW, Stoll BJ. Neonatal infectious diseases: evaluation of neonatal sepsis. Pediatr Clin North Am. 2013; 60:367-89. doi: 10. 1016/j.pcl.2012.12.003

13. Bizzarro MJ, Raskind C, Baltimore RS, Gallagher PG. Seventy-five years of neonatal sepsis at Yale: 1928-2003. Pediatrics. 2005 Sep;116(3):595-602.

14. Hornik CP, Fort P, Clark RH, Watt K, Benjamin DK Jr, Smith PB, Manzoni P, Jacqz-Aigrain E, Kaguelidou F, Cohen-Wolkowiez M. Early and late onset sepsis in very-low-birth-weight infants from a large group of neonatal intensive care units. Early Hum Dev. 2012 May;88 Suppl 2:S69-74. doi: 10.1016/ S0378-3782(12)70019-1.

15. Gerdes JS. Diagnosis and management of bacterial infections in the neonate. Pediatr Clin North Am. 2004 Aug; 51(4):939-59, viii-ix.

16. Bonadio WA, Hennes H, Smith D, Ruffing R, Melzer-Lange M, Lye P, Isaacman D. Reliability of observation variables in distinguishing infectious outcome of febrile young infants. Pediatr Infect Dis J. 1993;12:111-4. doi: 10.1097/00006454-19930200000001 .

17. Gerdes JS. Clinicopathologic approach to the diagnosis of neonatal sepsis. Clin Perinatol. 1991 Jun;18(2):361-81.

18. Gleason CA, Devaskar SU, Avery ME. Avery's diseases of the newborn / [edited by] Christine A. Gleason, Sherin U. Devaskar. Philadelphia, PA: Elsevier/Saunders, 2012.

19. Voora S, Srinivasan G, Lilien LD, Yeh TF, Pildes RS. Fever in full-term newborns in the first four days of life. Pediatrics. 1982 Jan;69(1):40-4. 
20. Weisman LE, Stoll BJ, Cruess DF, Hall RT, Merenstein GB, Hemming VG, Fischer GW. Earlyonset group B streptococcal sepsis: a current assessment. J Pediatr. 1992 Sep;121(3):428-33.

21. Hofer N, Müller W, Resch B. Neonates presenting with temperature symptoms: role in the diagnosis of early onset sepsis. Pediatr Int. 2012 Aug;54(4):486-90. doi: 10.1111/j.1442-200X.2012.03570.x. Epub 2012 Apr 9.

22. Bekhof J, Reitsma JB, Kok JH, Van Straaten IH. Clinical signs to identify late-onset sepsis in preterm infants. Eur J Pediatr. 2013 Apr;172(4):501-8. doi: 10. 1007 /s00431-012-1910-6. Epub 2012 Dec 28.

23. Remington JS. Infectious diseases of the fetus and newborn infant. Philadelphia, PA: Saunders/Elsevier, 2011.
24. Martin RJ, Fanaroff AA, Walsh MC. Fanaroff and Martin's neonatal-perinatal medicine: diseases of the fetus and infant. Philadelphia: Saunders/Elsevier, 2011.

25. Jean-Baptiste N, Benjamin DK, Jr, CohenWolkowiez M, et al. Coagulase-negative staphylococcal infections in the neonatal intensive care unit. Infect Control Hosp Epidemiol. 2011;32:679-686.

26. Stoll BJ, Hansen N, Fanaroff AA, et al. Late-onset sepsis in very low birth weight neonates: the experience of the NICHD Neonatal Research Network. Pediatrics. 2002;110 (2 Pt 1):285-291

27. Stoll BJ, Hansen NI, Bell EF, et al. Neonatal outcomes of extremely preterm infants from the NICHD Neonatal Research Network. Pediatrics. 2010;126:443-456.

\section{How to cite this article?}

Mahantesh. S, Manasa.S, Niranjan.H.S. Incidence of meningitis in blood culture proven neonatal sepsis in a pediatric tertiary care hospital in Bangalore. Trop J Path Micro 2017;3(2):206-212.doi: 10.17511/jopm.2017.i2.23. 